\title{
Genome-wide association study and Mendelian randomization analysis provide insights for improving rice yield potential
}

\section{Jing Su}

Hunan Agricultural University https://orcid.org/0000-0003-4272-4974

\section{Kai Xu}

Hunan Agricultural University

Chao Wu

Hunan Agricultural University

\section{Zirong Li}

Hunan Agricultural University

\section{Zhongli Hu}

Wuhan University

\section{Xingfei Zheng}

Hubei Academy of Agricultural Sciences

\section{Shufeng Song}

hunan hybird rice research center

\section{Wenbang Tang}

Hunan Agricultural University

\section{Zhonghai Tang}

Hunan Agricultural University

Lanzhi Li ( $\sim$ lancy0829@163.com)

https://orcid.org/0000-0002-1531-8500

\section{Original article}

Keywords: Rice yield, Component traits, Meta-GWAS, Mendelian randomization

Posted Date: July 24th, 2020

DOl: https://doi.org/10.21203/rs.3.rs-28492/v2

License: (c) (1) This work is licensed under a Creative Commons Attribution 4.0 International License.

Read Full License 


\section{Abstract}

Background: Rice yield has a complex genetic architecture, which mainly determined by its three component traits: the number of grains per panicle (GPP), kilo-grain weight (KGW) and tillers per plant (TP). Exploring ideotype breeding based on selection for genetically less complex component traits is an alternative route for further improving rice production. Thus, it is important that studying the genetic basis of relationship between rice yield and component traits and selecting the component traits to improve the rice production.

Main text: In this study, we carried out meta-analyses of genome-wide association study (Meta-GWAS) with two populations $\left(575+1495 \mathrm{~F}_{1}\right)$ in different environments for yield and its three component traits in rice. 3589 significant loci for three components traits were detected, while only 3 significant loci for yield were detected. It indicated that rice yield is mainly controlled by minor-effect loci and hardly to be identified. Selecting quantitative trait locus (QTL)/gene affected component traits to further enhance yield is recommended. Mendelian randomization (MR) design was adopted to investigate the genetic effects of loci on yield through component traits and estimate the genetic relationship between rice yield and its component traits by these loci. The loci for GPP or TP mainly had a positive genetic effect on yield, but the loci for KGW with different direction of effects (positive effect or negative effect). Additionally, TP (Beta=1.865) has a greater effect on yield than KGW (Beta=1.016) and GPP (Beta=0.086). Five significant loci for component traits with indirect effect on yield were identified. Pyramiding superior alleles of the five loci revealed improved yield. A combination of direct and indirect effects may better contribute to the yield potential of rice.

Conclusions: Our findings provided a rationale for using component traits as indirect indices to enhanced rice yield, which will be helpful for further understanding the genetic basis of yield, and provide valuable information for improving rice yield potential.

\section{Background}

Rice is a staple food crop for about half of world people. Improving rice productivity has been the main goal of rice breeding research since the growth of population and the loss of arable land. However, rice yield has a complex genetic architecture, which determined by various physiological processes changing temporally during the growing period. These processes often matched the yield component traits that are genetically less complex than yield (Kadam et al. 2018). Therefore, selecting the component traits of yield was proposed as a complementary route for further improving the rice production, which also has been emphasized by national and international rice breeding programs (Huang et al. 2009). Studying the genetic relationship between rice yield and component traits, and selecting the component traits to improve rice yield, will provide new clues for enhancing rice yield potential.

Rice yield is a very complex agronomic trait mainly determined by its three component traits: the number of grains per panicle (GPP), kilo-grain weight (KGW) and tillers per plant (TP), which are typical 
quantitative traits that are affected by multiple genes and the environment, with low heritability (Xing et al. 2010). With the development of high-throughput technology, a large number of genes/quantitative trait loci (QTLs) of the three component traits were identified using QTL mapping and genome-wide association study (GWAS) methods (Le et al. 2019; Wang et al. 2019). At the end of 2019, 209, 223, and 239 genes/QTLs for GPP (TO: 0000445), KGW (TO: 0000382), and TP (TO: 0000152) were identified respectively (http://www.gramene.org/), which densely distributed across the 12 chromosomes. Some of them have been applied in rational design of super rice by marker-assisted selection (MAS) breeding, in which multiple defined genes with superior alleles pyramided to increase rice yield (Qian et al. 2016). Liu et al. (2012) introduced the DEP1 and Gn1 genes introduced into the restorer line 93-11, then the yield of the DEP1 / Gn1-9311 line was significantly improved, due to resource allocation improved. In 2020, Wang et al. (2020) compared the transgenic lines with GNP1 or NAL 1 to the transgenic lines with both genes. They found the latter had a significantly higher yield, which indicated the two genes combinations may enhance the source-sink relationship. In the above researches, only a small number of genes combined for super rice breeding, if more genes are selected for pyramiding, the trade-offs between different traits need to carefully consider (Zeng et al. 2017). Therefore, understanding the nature and strength of the relationship between yield and its components will be helpful for efficient gene selection in MAS breeding (Li et al. 2019).

The relationship between rice yield and its components investigated by various researchers with different materials and methods, but they were inconsistent. In Huang et al.'s study, the superior alleles of grain number generally had a positive effect on yield, while the superior alleles of grain weight generally had a negative effect on yield (Huang et al. 2015). Path analyses were performed by Oladosu et al. (2018) on rice yield and component traits revealed that three component traits possessed positive effect with yield. Xu et al. (2015) conducted a correlation analysis between yield and its components of 300 rice germplasms. Their result indicated that yield was significantly correlated with GPP or KGW, but nonsignificant correlations of yield were found with TP. One possible explanation for the conflicting results is that the bias caused by the small sample size and lack of proper control for potential unmeasured confounders. Meta-analysis of GWAS is powerful in dissecting complex human diseases. It is the statistical synthesis of information from multiple cohorts independent GWAS studies, which increases power and reduces false-positive findings (Evangelou et al. 2013). Compared to humans, plants were planted in multiple years, environments, and locations. Meta-analysis is a useful way to narrowing down confidence intervals of QTL by compiling QTL information from multiple years, environments, and locations (Lzquierdo et al. 2018). A recent meta-analysis of GWAS in tomato demonstrated the benefits obtained from meta-analysis in plants. Meta-analysis can assess the heterogeneity of studies, which can be caused by many factors, such as phenotypic structure (data coming from different studies with different plant growth conditions, different harvesting and sampling procedures etc.), genetic structure (such as population structure and kinship), linkage disequilibrium, imputation accuracies or G×E interactions (Zhao et al. 2019).

Recently, Mendelian randomization (MR) approach is a popular technique to assess the causal relationship between disease and environmental risk factors within a meta-analysis framework in 
epidemiology (Bowden et al. 2019). MR method was used to investigate the role of ATP citrate lyase inhibitors in cardiovascular disease (Ference et al. 2019), in which the potential unmeasured confounders could be well protected from the observed association. In the MR approach, genetic variants were used as instrumental variables to avoid the possibility of confounding, because the genetic variants are randomly allocated at meiosis (Mokry et al. 2015). Thus, combine meta-analysis and MR for complex traits will help researchers to obtain a more reliable conclusion of their genetic relationship and further understand genetic basis of rice yield.

GWAS has been proved to be a new strategy for explaining the genetic basis of complex traits, which has the advantage of improving the efficiency of detecting natural variations (Li et al. 2018). Most GWAS studies focused on dissecting the genetic basis of single yield traits (Ta et al. 2018; Jiang et al. 2019), but the study on clarifying the genetic basis of the relationship between the yield and component traits of rice is few. Here we carried out meta-analyses of GWAS results from two populations $\left(575+1495 \mathrm{~F}_{1}\right)$ in different environments, and adopted an MR design to further estimated the causal relationship between rice yield and component traits of rice. Our aim was to detect significant single-nucleotide polymorphisms (SNPs) associated with yield or component traits, to analyze the genetic bases contributing to the relationship between them, and to investigate possible utilization pattern for selecting the component traits of yield in breeding practice so as to further understand to genetic basis of yield and improve the rice production. The study will provide theoretical guidelines for enhancing rice yield potential.

\section{Methods}

\section{Materials and phenotyping}

Two populations of rice hybrid varieties were used in our study. One of the populations consists of $575 \mathrm{~F}_{1}$ hybrid rice lines, which produced by 115 varieties (restorer lines of 29 three-line wild-deficient hybrid rice and 86 accessions of micro-core germplasm) as male parents were crossed with 5 sterile lines (4 two-line sterile lines and 1 three-line sterile line) as female parents. The 575 hybrid lines were grown both in Huazhong Agricultural University and Wuhan University in 2012. The other population from the national center for gene research of Chinese academy of sciences, which including 1,170 lines were bred from the three-line system and 325 lines were generated from the two-line system. The 1495 hybrid lines were grown in Hangzhou and Sanya respectively (Huang et al. 2015). Genotypic and phenotypic data of the 1495 lines were downloaded for subsequent analysis in this study. A total of four agronomic traits including GPP, KGW, TP, and yield (YD) were recorded in both populations. The phenotyping standards for these agronomic traits are the same in both populations, which were measured for at least three samples of each accession, and the average measurement was taken as the phenotypic value for GWAS analysis. Among them, KGW was initially obtained by weighing some grains, which was then converted to 1000 grain weight value, TP was obtained by measuring the number of panicles per plant.

\section{Resequencing and genotyping}


The population of 575 hybrid rice lines was sequenced on the Illumina HiSeq2500 platform at 11' genome coverage on average. By quality control, we obtained 1,894,012 high quality SNPs with minor allele frequency (MAF) $>5 \%$ and missing rate $<20 \%$ across the 575 accessions. The high diversity SNP maps of 1,495 hybrid rice varieties are publicly available (http://www.ncgr.ac.cn/RiceHap4) (Huang et al. 2015). The genomes of 1,495 hybrid lines were sequenced on the lllumina HiSeq2000 at twofold genome coverage, and 1,531,463 SNPs passing quality control (MAF >1\%).

\section{Genotype imputation and GWAS analysis}

3000 rice genomes project (https://snp-seek.irri.org/download.zul) as the reference panel was used to perform SNP imputation in the genotype data of 575 and 1495 hybrid rice lines by using beagle software (version 5.0) (Browning et al. 2018), and all imputed SNPs with MAF $<1 \%$ were filtered. Among the 3000 rice genomes project, the $4.8 \mathrm{mio}$ filtered SNP dataset is used as the reference panel in the study, with an average sequencing depth of $14^{\prime}$ and all SNP passed the quality control (MAF $>1 \%$, missing rate $=0 \%$ ) ( $\mathrm{Li}$ et al. 2014). Then conducting separate GWAS for two populations in two different environments using mixed-linear-model Association (MLMA) in GCTA software (Yang et al. 2014) and collecting the summary statistics to run a meta-GWAS.

\section{Meta-GWAS analyses}

Meta-GWAS is a meta-analysis of summary data (beta, standard error and $p$-values of each SNP) from each GWAS results. Imputation increased the genome-wide SNP densities, a total of 1,838,525 common SNPs from four GWAS datasets were used for meta-analysis. We used the fixed-effect model in METAL as the primary approach to conduct the meta-analyses (Willer et al. 2010). The fixed-effect model adopts the inverse variance weighting method, which weighted each study according to the inverse of its squared standard error. Then the Cochran's Q-test was performed to heterogeneity test (Cochran et al. 1954). For those SNPs where heterogeneity occurs $\left(R_{>}>50 \%\right)$, the random effect model in METASOFT was adopted (Han et al. 2011). The genome-wide significant $P$-value for meta-GWAS was set as $P<1 \mathrm{E}-06\left(-\log _{10} P=6\right)$.

\section{MR analysis}

For the genetic effect of rice yield and each component trait to be consistently estimated, the genetic variants were selected according to the three assumptions in MR analysis (Burgess et al. 2013), (i) the genetic variants were obtained from the results of meta-GWAS that associated with the single component trait at a genome-wide significant level $(P<1 \mathrm{E}-06)$; (ii) the genetic variants are not associated with any confounders; (iii) the genetic variants only affect yield through the single component trait, not through other component traits ( $P>0.05)$. Since the selected SNPs in linkage disequilibrium (LD) may result in confounding (Mokry et al. 2015), we calculated the $r^{2}$ (LD) of all pairs between all selected SNPs using plink (version 1.90) (Chang et al. 2015) and discarded SNPs in LD $\left(r^{2}>0.01\right)$ based on larger $P$-value.

The inverse-variance weighting (IVW) method was conducted for MR analysis to assess the effect of component traits on yield, which by summarizing the effects of multiple independent SNPs (Burgess et 
al. 2013). In sensitivity analyses, the weighted median method (Bowden et al. 2016) and MR-Egger method (Burgess et al. 2017) are used for MR analysis, which is more robust due to pleiotropic or invalid instruments involved.

\section{Analysis of superior alleles of significant associate loci}

Calculated the average phenotypic measurement corresponding to genotypes of each significant SNP, and the least significant difference method was used for multiple comparisons. Following Huang et al.'s method (Huang et al. 2015), the genotype of SNP with the highest-level yield or component trait was set to be the superior allele (for example, the allele corresponding to the largest number of grains per panicle was set to be the superior allele). Calculated the number of superior alleles in each hybrid rice line and recorded their corresponding average yield measurements. Omitted the number of superior alleles with less than 3 hybrid lines.

\section{Results}

\section{Meta-GWAS analyses}

Meta-analyses of GWAS were performed based on four datasets' (two locations for each population) GWAS results (Additional file 1: Figures S1-S4). Manhattan plots and quantile-quantile plots of metaGWAS are shown in Fig. 1. A total of 3592 significant loci were identified (Additional file 2: Table S1), including 2450, 1116, 23 and, 3 significant associated loci were separately detected for GPP, KGW, TP, and YD, which were distributed on all of the rice chromosomes except for chromosome 10. According to the information of RAP-DB (http://rapdb.dna.affrc.go.jp/), candidate genes were searched in a genomic region of 200KB around the associated SNPs (Additional file 1: Table S2). We discovered 7, 7 and 3 cloned genes separately associated with GPP, KGW and TP. A total of three candidate genes associated with different traits, among which OsBZR1 (Zhu et al. 2015) and OSSPL 14 (Jiao et al. 2010) have been reported previously, and OSO2g0106966 was novelty discovered. Both OSBZR1 and OSSPL 14 were detected in KGW and GPP, Os02g0106966 was detected in KGW and TP. In this study, only 3 significant loci for YD were detected, but 3589 significant loci for the component traits were detected. It may be because rice yield has a low heritability which mainly affected by many minor-effect genes, the low heritability of rice yield is also showed in our previous study (Xu et al. 2018). These results suggested that selecting the component traits of yield as a complementary route to improve rice production is recommended.

\section{The genetic relationship between GPP and YD}

As required for MR analysis, a total of 2450 SNPs reached genome-wide significance for GPP $(P<1 \mathrm{E}-06)$ in meta-analyses of GWAS, among which six SNPs were selected as instrumental variables to estimate the genetic relationship between GPP and YD (Table 1). The six SNPs were not associated with KGW or TP $(P>0.05)$, and no evidence of LD between them (all pairwise $\left.r^{2}<0.01\right)$. In MR analysis, these loci mainly had a positive genetic effect on yield through GPP and a positive genetic relationship between GPP and 
YD were observed with the IVW method (Table 4, Fig. 2a). One standard deviation (SD) genetic higher GPP was associated with a 0.086 SD higher YD (Beta $=0.086,95 \% \mathrm{Cl}: 0.030 \sim 0.141, P=0.003)$. In sensitivity analyses, the Cochran's Q-test illustrated no obvious heterogeneity $\left(P^{2}=5 \%, P=0.38\right)$. The weighted median method also showed GPP had a positive effect on YD (Beta $=0.081,95 \% \mathrm{Cl}$ : $0.009 \sim 0.152, P=0.028)$. MR-Egger regression indicated no evidence of directional pleiotropy for the associations of GPP with YD (intercept $=1.387 \otimes P=0.061$ ). It is worth noting that some cloned genes were detected in the meta-GWAS on GPP, the phenotype of transgenic plants with these genes had a similar phenomenon. For example, the OSSPL14 mutant produced more grain number per panicle, enhanced rice yield (Jiao et al. 2010). Compared with the control non-transgenic plants, the over-expression of OsBZR1 plants showed the 1000-grain weight was increased by about $3.4 \%$ and the spikelet number per panicle was increased $21.9 \%$, that resulting in enhanced yield (Zhu et al. 2015). The $c d 1$ mutant exhibited a variety of phenotypic traits, such as a reduction in grain number and panicle length, the biomass was lower than that of the wild type (Luan et al. 2011).

Table 1 Information about instrumental variables

\begin{tabular}{ccccccc}
\hline \multirow{2}{*}{ SNP } & Chromosome & Position & \multicolumn{2}{c}{ GPP } & \multicolumn{2}{c}{ YD } \\
\cline { 4 - 6 } & & & Beta & $P$-value & Beta & $P$-value \\
\hline chr03_29979498 & 3 & 29979498 & -18.724 & $1.12 \mathrm{E}-07$ & -0.633 & 0.543 \\
chr03_898774 & 3 & 898774 & 18.953 & $2.92 \mathrm{E}-07$ & 1.254 & 0.299 \\
\hline chr05_7226049 & 5 & 7226049 & -7.242 & $2.25 \mathrm{E}-08$ & -1.329 & 0.005 \\
\hline chr08_25257522 & 8 & 25257522 & -16.559 & $8.58 \mathrm{E}-07$ & 1.178 & 0.553 \\
\hline chr09_12464309 & 9 & 12464309 & -7.538 & $4.49 \mathrm{E}-07$ & -0.974 & 0.066 \\
\hline chr12_22633431 & 12 & 22633431 & 15.738 & $3.01 \mathrm{E}-07$ & 1.439 & 0.158 \\
\hline
\end{tabular}

Note: All the SNP markers are named after the chromosome _ position

\section{The genetic relationship between KGW and YD}

As required for MR analysis, a total of 1116 SNPs reached genome-wide significance for KGW $(P<1 \mathrm{E}-06)$ in meta-analyses of GWAS, among which eleven SNPs were selected as instrumental variables to estimate the genetic relationship between KGW and YD (Table 3). These SNPs were not associated with GPP or TP $(P>0.05)$, and no evidence of LD between them (all pairwise $\left.r^{2}<0.01\right)$. In MR analysis, we observed that a part of SNP for KGW had a positive effect on YD, a part of SNP for KGW had a negative effect on YD (Table 2, Fig. 2b). To further understand to genetic relationship between KGW and YD, the SNPs with different direction of genetic effects are studied separately in our study. These loci with positive effect showed that KGW had a positive effect on yield, while these loci with negative effect showed that KGW had no significant negative effect on yield (Fig. 3). In sensitivity analyses, the Cochran's Q-test illustrated no obvious heterogeneity $\left(R^{2}=0 \%\right)$. The weighted median method also confirmed the results of the IVW method. MR-Egger regression indicated no evidence of pleiotropy for the 
associations of KGW with YD (Table 4). The cloned gene GW2 was detected in the meta-GWAS on KGW has been reported have the potential to enhance rice yield (Song et al. 2007).

Table 2 Information about instrumental variables

\begin{tabular}{|c|c|c|c|c|c|c|}
\hline \multirow[t]{2}{*}{ SNP } & \multirow[t]{2}{*}{ Chromosome } & \multirow[t]{2}{*}{ Position } & \multicolumn{2}{|c|}{ KGW } & \multicolumn{2}{|c|}{ YD } \\
\hline & & & Beta & $P$-value & Beta & $P$-value \\
\hline chr01_3547491 & 1 & 3547491 & 0.706 & $7.14 \mathrm{E}-08$ & -0.036 & 0.948 \\
\hline chr01_5524333 & 1 & 5524333 & 1.018 & $6.56 \mathrm{E}-07$ & 0.310 & 0.840 \\
\hline chr02_1118809 & 2 & 1118809 & 0.786 & $1.56 \mathrm{E}-09$ & 1.030 & 0.082 \\
\hline chr02_334316 & 2 & 334316 & -1.474 & $2.38 \mathrm{E}-07$ & 0.948 & 0.505 \\
\hline chr02_7792121 & 2 & 7792121 & -1.256 & $9.21 \mathrm{E}-08$ & 0.029 & 0.980 \\
\hline chr03_17810847 & 3 & 17810847 & 0.651 & $4.74 \mathrm{E}-07$ & 0.711 & 0.394 \\
\hline chr03_33060865 & 3 & 33060865 & -0.505 & $2.03 \mathrm{E}-08$ & -0.978 & 0.027 \\
\hline chr04_13785932 & 4 & 13785932 & 0.739 & $3.16 \mathrm{E}-07$ & 0.623 & 0.406 \\
\hline chr05_16393143 & 5 & 16393143 & 0.466 & $4.71 \mathrm{E}-07$ & 0.029 & 0.955 \\
\hline chr07_23215227 & 7 & 23215227 & 0.952 & $1.09 \mathrm{E}-07$ & -0.308 & 0.715 \\
\hline chr08_25464238 & 8 & 25464238 & -0.875 & $2.01 \mathrm{E}-09$ & -0.004 & 0.997 \\
\hline
\end{tabular}

\section{The genetic relationship between TP and YD}

As required for MR analysis, a total of 23 SNPs reached genome-wide significance for TP $(P<1 \mathrm{E}-06)$ in meta-analyses of GWAS, among which three SNPs were selected as instrumental variables to estimate the causal relationship between TP and YD (Table 3). These SNPs were not associated with KGW or GPP $(P>0.05)$, and no evidence of LD between them (all pairwise $r^{2}<0.01$ ). In MR analysis, these loci had a positive genetic effect on yield through TP and a positive causal relationship between TP and YD were observed with the IVW method (Table 4, Fig. 2c), 1 SD genetic higher TP was associated with a 1.865 SD higher YD (Beta=1.865, 95\% Cl: 1.035 2.694, $P<0.0001)$. Compared with KGW (Beta=1.016) and GPP (Beta=0.086), TP (Beta=1.865) has a greater effect on yield. In sensitivity analyses, Cochran's Q-test illustrated no obvious heterogeneity $\left(P^{2}=0 \%, P=0.43\right)$. The weighted median method also showed TP had a positive effect on YD (Beta=1.54, 95\% Cl: 0.353 2.727, $P=0.011)$. MR-Egger regression indicated no evidence of directional pleiotropy for the associations of TP with YD (intercept=0.046, $P=0.968$ ). The cloned gene OsPIN2 was detected in the meta-GWAS on TP. Chen et al. (2012) found that the OsPIN2 transgenic plants had more effective tiller number, lower 1000-grain weight, and higher yield.

Table 3 Information about instrumental variables 


\begin{tabular}{|c|c|c|c|c|c|c|}
\hline \multirow[t]{2}{*}{ SNP } & \multirow[t]{2}{*}{ Chromosome } & \multirow[t]{2}{*}{ Position } & \multicolumn{2}{|c|}{$\mathrm{TP}$} & \multicolumn{2}{|c|}{ YD } \\
\hline & & & Beta & $P$-value & Beta & $P$-value \\
\hline chr02_21604477 & 2 & 21604477 & -1.279 & 8.25E-07 & -1.977 & 0.043 \\
\hline chr06_1578700 & 6 & 1578700 & -0.639 & $4.52 \mathrm{E}-07$ & -1.691 & $3.21 \mathrm{E}-04$ \\
\hline chr11_26492375 & 11 & 26492375 & -0.507 & 7.04E-08 & -0.720 & 0.043 \\
\hline
\end{tabular}

Table 4 MR results of the relationship between yield and its component traits

\begin{tabular}{|c|c|c|c|c|}
\hline Trait & Methods & Beta & $95 \% \mathrm{CI}$ & $P$ \\
\hline \multirow[t]{4}{*}{ GPP } & IVW & 0.086 & $0.030 \sim 0.141$ & 0.003 \\
\hline & Weighted median & 0.081 & $0.009 \sim 0.152$ & 0.028 \\
\hline & MR-Egger & -0.029 & $-0.160 \sim 0.103$ & 0.668 \\
\hline & MR-Egger(intercept) & 1.387 & $-0.063 \sim 2.836$ & 0.061 \\
\hline \multirow[t]{4}{*}{ KGW (positive) } & IVW & 1.016 & $0.242 \sim 1.791$ & 0.010 \\
\hline & Weighted median & 1.123 & $0.122 \sim 2.124$ & 0.028 \\
\hline & MR-Egger & 0.480 & $-2.743 \sim 3.704$ & 0.770 \\
\hline & MR-Egger(intercept) & 0.349 & $-1.690 \sim 2.388$ & 0.737 \\
\hline KGW & IVW & -0.233 & $-1.092 \sim 0.626$ & 0.595 \\
\hline \multirow[t]{3}{*}{ (negative) } & Weighted median & -0.156 & $-1.150 \sim 0.839$ & 0.759 \\
\hline & MR-Egger & -0.710 & $-3.853 \sim 2.434$ & 0.658 \\
\hline & MR-Egger(intercept) & 0.464 & $-2.480 \sim 3.407$ & 0.757 \\
\hline \multirow[t]{4}{*}{$\mathrm{TP}$} & IVW & 1.865 & $1.035 \sim 2.694$ & $<0.0001$ \\
\hline & Weighted median & 1.540 & $0.353 \sim 2.727$ & 0.011 \\
\hline & MR-Egger & 1.797 & $-1.633 \sim 5.228$ & 0.304 \\
\hline & MR-Egger(intercept) & 0.046 & $-2.165 \sim 2.256$ & 0.968 \\
\hline
\end{tabular}

Note: $C l$ confidence intervals, $P$ statistically significant associations with a $P<0.05$.

\section{Loci for component traits had an indirect effect on yield}

We identified five significant loci that had an indirect effect on yield by MR analyses (Fig. 2, Additional file 1: Table S3). Among them, the SNP chr05_7226049 (Fig. 2a) for GPP had an indirect effect on yield and located nearby the cloned gene OSPYL11. Kim et al. (2014) reported that compared with the control plants, the transgenic plants overexpressing OSPYL11 showed no significant difference in tiller number, 
but the yield was severely reduced. Our study indicated the yield severely reduced may be caused by the number of grains decreased. The SNP chr03_33060865 (Fig. 2b) for KGW is in the vicinity of the cloned gene $E L 1$, which is a key regulator of the gibberellin response, Kwon et al. (2015) discovered the plants that loss of EL 1 showed the 500-grain weight and yield significantly reduced. The SNP chr06_1578700 (Fig. 2c) for TP closed to the D62 (a gene regulating tillers). Li et al. (2010) found that the tiller number of D62 mutant rice was less than that of the wild type. The SNPs chr02_21604477 and chr11_26492375 for TP also had indirect effects on yield (Fig. 2c, Table S3), which were first detected in our research. These findings provided new information for further improve rice yield potential.

\section{Pyramiding superior alleles of significant loci}

The average yield performance of $F_{1}$ lines with different superior allele numbers of significant loci with direct effect, indirect effect, and direct plus indirect effect were shown in Fig. 4. Three loci had a direct effect on yield were detected in the meta-GWAS on YD (Additional file 1: Table S3), among them, the average yield of the lines without superior alleles was $41.29 \mathrm{~g}$, and the average yield of the lines with one superior allele was $44.26 \mathrm{~g}$ (Fig. 4a, Additional file 1: Table S4). The superior alleles of five loci that had an indirect effect on yield were also pyramided in the study. The results showed that the average yield of $F_{1}$ lines with 0 to 4 superior alleles was: $42.22 \mathrm{~g}, 42.75 \mathrm{~g}, 42.76 \mathrm{~g}, 44.54 \mathrm{~g}, 47.49 \mathrm{~g}$, respectively. In general, the yield of $F_{1}$ hybrids rises with increases of the superior alleles (Fig. $4 \mathrm{~b}$, Additional file 1: Table S4). A similar phenomenon also found in pyramiding the direct plus indirect loci (Fig. 4c, Additional file 1: Table S4). Other research reported that the phenotype performance improved by pyramiding the superior alleles of loci associated with agronomic traits (Huang et al. 2015), our results suggested the yield enhanced also by pyramiding the superior alleles of loci that had an indirect effect on yield. Hybrid lines pyramiding all the superior alleles of direct (3 loci) and indirect loci (5 loci) not be observed in this study. Our results indicated rice production improved with increased of the superior alleles, it is possible that a combination of direct and indirect effects will better contribute to the yield potential of rice.

\section{Discussion}

In this study, a total of 3592 significant SNPs were detected in meta-GWAS on yield or its component traits, which provide more information for rice agronomic traits breeding. It is worth noting that only 3 loci were detected in meta-GWAS on yield, this may be results from that rice yield has a low heritability and minor-effect loci hardly to be detected. For a low-heritability trait (such as yield), highly correlated auxiliary traits (such as GPP) will help improve the selection of traits with low heritability since they reflecting a shared biological basis (Wang et al. 2017).

MR model was carried out to investigate the genetic effects of loci on yield through component traits. The loci for GPP or TP mainly had a positive genetic effect on yield, which was consistent with previous studies (Jiao et al. 2010; Chen et al. 2012). The loci for KGW had different direction of effects (positive effect or negative effect) on yield. Huang et al. (2015) conducted GWAS on 1495 hybrid rice lines and found the superior alleles of grain weight generally had a negative effect on yield, but some genes that 
regulate KGW have been reported have the potential to enhance rice yield, such as GW7 (Wang et al. 2015) and GS5 (Li et al. 2011). In some extent, our results confirmed both of their findings, which indicated our study had greater power and more comprehensive by synthesizes different data. Then these loci were used to explore the relationship between rice yield and its component traits through these loci by MR method. The results of MR provided some evidence that selecting the component traits of yield to improve rice production, which are consistent with the improvement of rice production achieved by genetic manipulation of the component traits in previous studies (Yan et al. 2011; Wang et al. 2015). The MR analyses provided a rationale for using component traits as indirect indices to enhanced rice yield.

Five loci were identified with an indirect effect on yield by MR analysis, providing new information for enhancing the yield potential of rice. A previous study indicated that pyramiding the superior alleles of significant associated loci increased yield (Huang et al. 2015). Our results suggested the improvement of yield also by pyramiding the superior alleles of loci with an indirect effect on yield. In this study, due to the limitation of sample size, hybrid lines pyramiding all the superior alleles of direct (3 loci) and indirect loci (5 loci) not be observed. However, it is interesting to note that the average yield performance of hybrid lines with 1 to 3 superior alleles of indirect loci improved with increased of the superior alleles, when adding a superior alleles of direct locus to pyramid, the average yield performance of hybrid lines were increased. Similar to the performance of the superior alleles of four indirect loci, the yield of the superior alleles of five direct plus indirect loci also be improved (Fig. 4). Our results indicated rice production improved with increased of the superior alleles, it is possible that a combination of direct and indirect effects will better contribute to the yield potential of rice.

The strengths of the study are: (i) a meta-analysis of GWAS data from multiple population and environments to estimate a summary effect provided greater statistical power (Panagiotou et al. 2013); (ii) Combining GWAS and MR for complex traits contributes to understand the genetic basis of complex traits (Shah et al. 2020); (iii) MR method was used to analyze the causal relationship between quantitative traits in this study, which weighted the effects of multiple independent SNPs into a summary effect, for quantitative traits, most of them are affected by multiple genes or the interaction of genes, while the individual SNP only explain a small fraction of the variation in the quantitative traits. Since the MR analysis may be biased by the possibility of invalid instrumental variables, it is difficult to completely exclude type I error and the potential influence of pleiotropy since the instrumental variables derived from the meta-analysis of GWAS in the study. Thus, we conducted a weighted median method and MR-Egger method to do sensitivity analysis. Compared to the IVW method, the weighted median method showed to have better finite-sample Type I error rates, the estimator is consistent even if up to $50 \%$ of the information comes from invalid instrumental variables (Bowden et al. 2016). The results of the MR-Egger and heterogeneity test indicated the genetic variants had no pleiotropic effects on yield to some extent (Burgess et al. 2017). These results strengthened our confidence in the validity of assumptions.

\section{Conclusion}


In conclusion, we analyzed the genetic basis of the relationship between yield and its component traits by GWAS and MR methods, providing genetic insights for further improving rice yield potential. Our results suggested the improvement of rice production by pyramiding the superior alleles of genes regulating component traits, and a combination of direct and indirect effects may better contribute to the yield potential of rice in breeding practice. These findings will provide theoretical guidelines for the rational design of rice by MAS breeding.

\section{Declarations}

\section{Ethics approval and consent to participate}

No applicable.

\section{Consent for publication}

No applicable.

\section{Availability of data and materials}

The datasets supporting the conclusions of this article are provided within the article and its additional files, the datasets are available from the corresponding author on reasonable request.

\section{Competing Interests}

The authors declare that they have no competing interests

\section{Funding}

This work was supported by funding from the National key technology research and development program (2016YFD0100101), Open Research Fund of State Key Laboratory of Hybrid Rice (Hunan Hybrid Rice Research Center (2019KF05); Wuhan University (KF201912)) , Research Foundation of Education Bureau of Hunan Province (19A244), Double first-class construction project of Hunan Agricultural University (SYL2019028), Hubei Provincial cooperative Innovation Center囚Hubei Science and Education letter (2016) No.3.

\section{Authors' contributions}

LL designed and supervised the research; JS, ZL, XZ, ZH, SS, WT and ZT performed the research; JS, KX and CW analyzed the data; JS and LL wrote the paper. All authors read and approved the final manuscript.

\section{Acknowledgements}

We thank Prof. Sibin Yu and Tongmin Mou from Huazhong Agricultural University, Prof. Shuangcheng Li from Sichuan Agricultural University, Prof. Shuzhu Tang from Yangzhou University, Prof. Guanghua He 
from Southwest University, Prof. Huaxiong Qi from Hubei Academy of Agricultural Sciences for providing material to us. We also gratefully thank Zhaoming Chen from Guangzhou Institutes of Biomedicine and Health, Chinese Academy of Sciences for supercomputing technology support. We thank Yuan Hu from Hunan Agricultural University for revising paper.

\section{Abbreviations}

GPP: the number of grains per panicle; KGW: kilo-grain weight; TP: tillers per plant; GWAS: genome-wide association study; QTL:quantitative trait locus; MAS: marker-assisted selection; MR: Mendelian randomization; SNP: single-nucleotide polymorphism; YD: yield; MAF: minor allele frequency; IVW: inverse-variance weighting; LD: linkage disequilibrium; Cl: confidence intervals; SD: standard deviation

\section{References}

1. Bowden J, Davey Smith G, Haycock PC, Burgess S (2016) Consistent estimation in Mendelian randomization with some invalid instruments using a weighted median estimator. Genetic epidemiology 40: 304-314

2. Bowden J, Del Greco MF, Minelli C, Zhao Q, Lawlor DA, Sheehan NA, Davey Smith G (2019) Improving the accuracy of two-sample summary-data Mendelian randomization: moving beyond the NOME assumption. International journal of epidemiology 48:728-742

3. Browning BL, Zhou Y, Browning SR (2018) A one-penny imputed genome from next-generation reference panels. The American Journal of Human Genetics 103: 338-348

4. Burgess S, Butterworth A, Thompson SG (2013) Mendelian randomization analysis with multiple genetic variants using summarized data. Genetic epidemiology 37:658-665

5. Burgess S, Thompson SG (2017) Interpreting findings from Mendelian randomization using the MREgger method. European journal of epidemiology 32:377-389

6. Chang CC, Chow CC, Tellier LC, Vattikuti S, Purcell SM, Lee JJ (2015) Second-generation PLINK: rising to the challenge of larger and richer datasets. Gigascience 4: s13742-015

7. Chen Y, Fan X, Song W, Zhang Y, Xu G (2012) Over-expression of OsPIN2 leads to increased tiller numbers, angle and shorter plant height through suppression of OsLAZY1. Plant biotechnology journal 10:139-149

8. Cochran WG (1954) The combination of estimates from different experiments. Biometrics 10: 101129

9. Evangelou E, loannidis JP (2013) Meta-analysis methods for genome-wide association studies and beyond. Nature Reviews Genetics 14: 379-389

10. Ference BA, Ray KK, Catapano AL, Ference TB, Burgess S, Neff DR, Danesh J (2019) Mendelian randomization study of ACLY and cardiovascular disease. New England Journal of Medicine 380:1033-1042 
11. Han B, Eskin E (2011) Random-effects model aimed at discovering associations in meta-analysis of genome-wide association studies. The American Journal of Human Genetics 88: 586-598

12. Huang X, Qian Q, Liu Z, Sun H, He S, Luo D, Fu,X (2009) Natural variation at the DEP1 locus enhances grain yield in rice. Nature genetics 41:494

13. Huang X, Yang S, Gong J, Zhao Y, Feng Q, Gong H, Chen N (2015) Genomic analysis of hybrid rice varieties reveals numerous superior alleles that contribute to heterosis. Nature communications 6:1-9

14. Izquierdo P, Astudillo C, Blair MW, Iqbal AM, Raatz B, Cichy KA (2018) Meta-QTL analysis of seed iron and zinc concentration and content in common bean (Phaseolus vulgaris $L$ ). Theoretical and Applied Genetics 131:1645-1658

15. Jiang S, Wang D, Yan S, Liu S, Liu B, Kang H, Wang GL (2019) Dissection of the Genetic Architecture of Rice Tillering using a Genome-wide Association Study. Rice 12: 43

16. Jiao Y, Wang Y, Xue D, Wang J, Yan M, Liu G, Qian Q (2010) Regulation of OsSPL14 by OsmiR156 defines ideal plant architecture in rice. Nature genetics 42: 541

17. Kadam NN, Struik PC, Rebolledo MC, Yin X, Jagadish, SK (2018) Genome-wide association reveals novel genomic loci controlling rice grain yield and its component traits under water-deficit stress during the reproductive stage. Journal of experimental botany 69: 4017-4032

18. Kim H, Lee K, Hwang H, Bhatnagar N, Kim DY, Yoon IS, Kim BG (2014) Overexpression of PYL5 in rice enhances drought tolerance, inhibits growth, and modulates gene expression. Journal of experimental botany 65: 453-464

19. Kwon CT, Kim SH, Kim D, Paek NC (2015) The rice floral repressor Early flowering1 affects spikelet fertility by modulating gibberellin signaling. Rice 8:23

20. Le Nguyen K, Grondin A, Courtois B, Gantet P (2019) Next-generation sequencing accelerates crop gene discovery. Trends in plant science 24:263-274

21. Li F, Xie J, Zhu X, Wang X, Zhao Y, Ma X, Zhang S (2018) Genetic basis underlying correlations among growth duration and yield traits revealed by GWAS in rice (Oryza sativa L). Frontiers in plant science 9: 650

22. Li JY, Wang J, Zeigler RS (2014) The 3000 rice genomes project: new opportunities and challenges for future rice research. Giga science 3: 2047-217X-3-8

23. Li R, Li M, Ashraf U, Liu S, Zhang J (2019) Exploring the relationships between yield and yield-related traits for rice varieties released in China from 1978 to 2017. Frontiers in plant science 10:543

24. Li W, Wu J, Weng S, Zhang Y, Zhang D, Shi C (2010) Identification and characterization of dwarf 62, a loss-of-function mutation in DLT/OsGRAS-32 affecting gibberellin metabolism in rice. Planta 232:1383-1396

25. Li Y, Fan C, Xing Y, Jiang Y, Luo L, Sun L, He Y (2011) Natural variation in GS5 plays an important role in regulating grain size and yield in rice. Nature genetics 43:1266-1269

26. Liu J, Chen J, Zheng X, Wu F, Lin Q, Heng Y, Zhang X (2017) GW5 acts in the brassinosteroid signalling pathway to regulate grain width and weight in rice. Nature Plants 3:1-7 
27. Liu J, Tao H, Shi S, Ye W, Qian Q, Guo L (2012) Genetics and breeding improvement for panicle type in rice. Chinese Journal of Rice Science 26: 227-234

28. Luan W, Liu Y, Zhang F, Song Y, Wang Z, Peng Y, Sun Z (2011) OsCD1 encodes a putative member of the cellulose synthase-like $D$ sub-family and is essential for rice plant architecture and growth. Plant biotechnology journal 9:513-524

29. Mokry LE, Ross S, Ahmad OS, Forgetta V, Smith GD, Leong A, Richards JB (2015) Vitamin D and risk of multiple sclerosis: a Mendelian randomization study. PLoS medicine 12(8)

30. Oladosu Y, Rafii MY, Magaji U, Abdullah N, Miah G, Chukwu SC, Kareem I (2018) Genotypic and phenotypic relationship among yield components in rice under tropical conditions. BioMed research international 2018:1-10

31. Qian Q, Guo L, Smith SM, Li J (2016) Breeding high-yield superior quality hybrid super rice by rational design. National Science Review 3:283-294

32. Song XJ, Huang W, Shi M, Zhu MZ, Lin HX (2007) A QTL for rice grain width and weight encodes a previously unknown RING-type E3 ubiquitin ligase. Nature genetics 39: 623-630

33. Ta KN, Khong NG, Ha TL, Nguyen DT, Mai DC, Hoang TG, Dinh BY (2018) A genome-wide association study using a Vietnamese landrace panel of rice (Oryza sativa) reveals new QTLs controlling panicle morphological traits. BMC plant biology 18:282

34. Wang Q, Tang J, Han B, Huang X (2019) Advances in genome-wide association studies of complex traits in rice. Theoretical and Applied Genetics 1-11

35. Wang S, Li S, Liu Q, Wu K, Zhang J, Wang S, Wang F (2015) The OsSPL16-GW7 regulatory module determines grain shape and simultaneously improves rice yield and grain quality. Nature genetics 47 : 949-954

36. Wang X, Li L, Yang Z, Zheng X, Yu S, Xu C, Hu Z (2017) Predicting rice hybrid performance using univariate and multivariate GBLUP models based on North Carolina mating design II. Heredity 118:302-310

37. Wang Y, Zhai L, Chen K, Shen C, Liang Y, Wang C, Xu J (2020) Natural Sequence Variations and Combinations of GNP1 and NAL1 Determine the Grain Number per Panicle in Rice. Rice 13:1-15

38. Willer CJ, Li Y, Abecasis GR (2010) METAL: fast and efficient meta-analysis of genomewide association scans. Bioinformatics 26:2190-2191

39. Xing Y, Zhang Q (2010) Genetic and molecular bases of rice yield. Annual review of plant biology 61:421-442

40. Xu Q, Chen W, Xu Z (2015) Relationship between grain yield and quality in rice germplasms grown across different growing areas. Breeding science 65:226-232

41. Xu Y, Wang X, Ding X, Zheng X, Yang Z, Xu C, Hu Z (2018) Genomic selection of agronomic traits in hybrid rice using an NCll population. Rice 11:1-10

42. Yan WH, Wang P, Chen HX, Zhou HJ, Li QP, Wang R, Zhang QF (2011) A major QTL, Ghd8, plays pleiotropic roles in regulating grain productivity, plant height, and heading date in rice. Molecular 
plant 4:319-330

43. Yang J, Zaitlen NA, Goddard ME, Visscher PM, Price AL (2014) Mixed model association methods: advantages and pitfalls. Nat Genet 46:100-106

44. Zeng D, Tian Z, Rao Y, Dong G, Yang Y, Huang L, Hu J (2017) Rational design of high-yield and superior-quality rice. Nature plants 3:1-5

45. Zhao J, Sauvage C, Zhao J, Bitton F, Bauchet G, Liu D, Causse M (2019) Meta-analysis of genomewide association studies provides insights into genetic control of tomato flavor. Nature communications 10:1-12

46. Zhu X, Liang W, Cui X, Chen M, Yin C, Luo Z, Zhang D (2015) Brassinosteroids promote development of rice pollen grains and seeds by triggering expression of Carbon Starved Anther, a MYB domain protein. The Plant Journal 82: 570-581

47. Zhu YJ, Sun ZC, Niu XJ, Ying JZ, Fan YY, Mou TM, Zhuang JY (2019) Dissection of three quantitative trait loci for grain size on the long arm of chromosome 10 in rice (Oryza sativa L). PeerJ 7:e6966

\section{Figures}
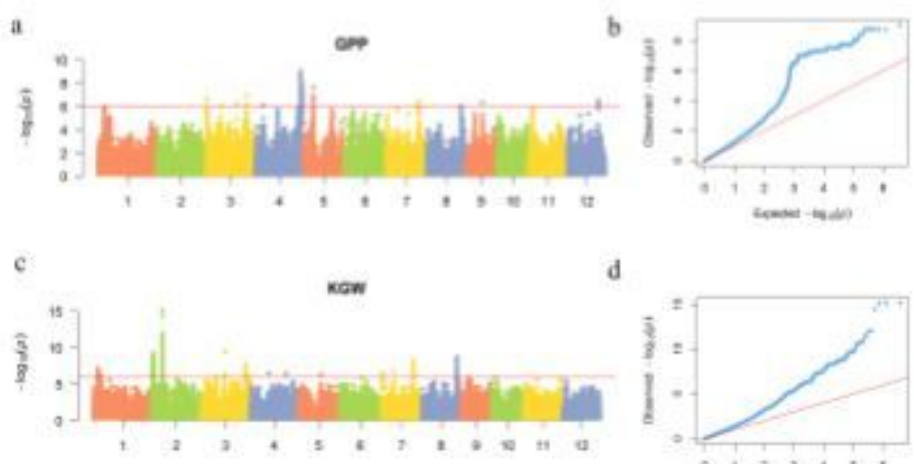

d
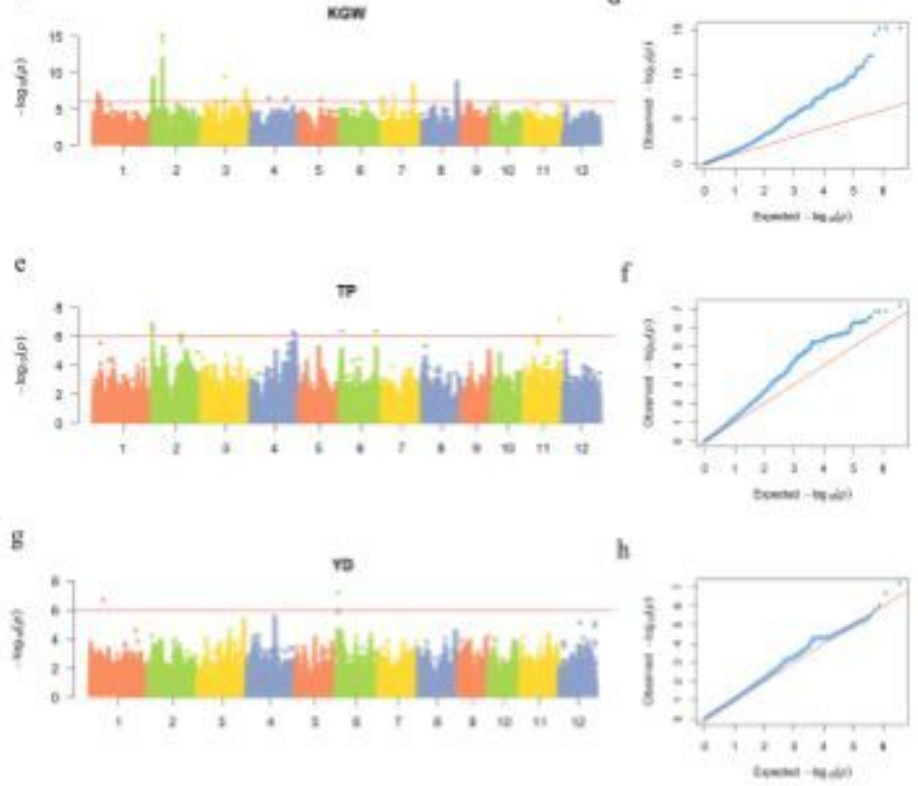

\section{Figure 1}

Meta-analyses results for GWAS. ( $a, b)$ Manhattan plots and quantile-quantile plots of GPP. (c, d) Manhattan plots and quantile-quantile plots of KGW. $(e, f)$ Manhattan plots and quantile-quantile plots 
of TP. $(\mathrm{g}, \mathrm{h})$ Manhattan plots and quantile-quantile plots of YD. The genome-wide significant P-value threshold $P<10-6$ is indicated by a horizontal line.

a

SNP
chr03_29979498
chr03_898774
chr05_7226049
chr08_25257522
chr09_12464309
chr12_22633431

Summary

c

\section{SNP}

chr02_21604477

chr06_1578700

chr11_26492375

Summary

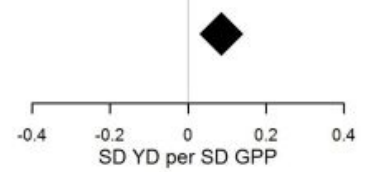

b

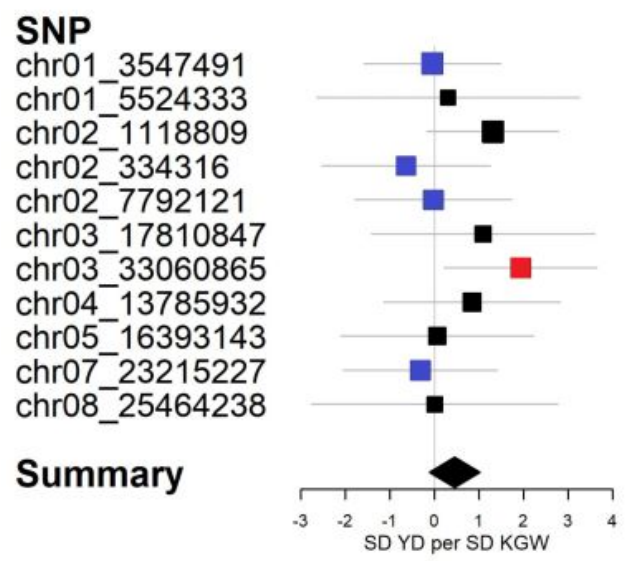

SNP

Chro2-334316

(1)

chr03 17810847

chr03 33060865

chr04 13785932

chr05-16393143

chr07 23215227

chros_25464238

Summary
- positive effect

$\square$ negative effect

$\square$ indirect effect

\section{Figure 2}

Genetic effect estimates of yield and its components. Estimates are derived from IVW method of MR analyses. a Effect estimates between GPP and YD. b Effect estimates between KGW and YD. c Effect estimates between TP and YD. 
a

$\mathrm{b}$

SNP

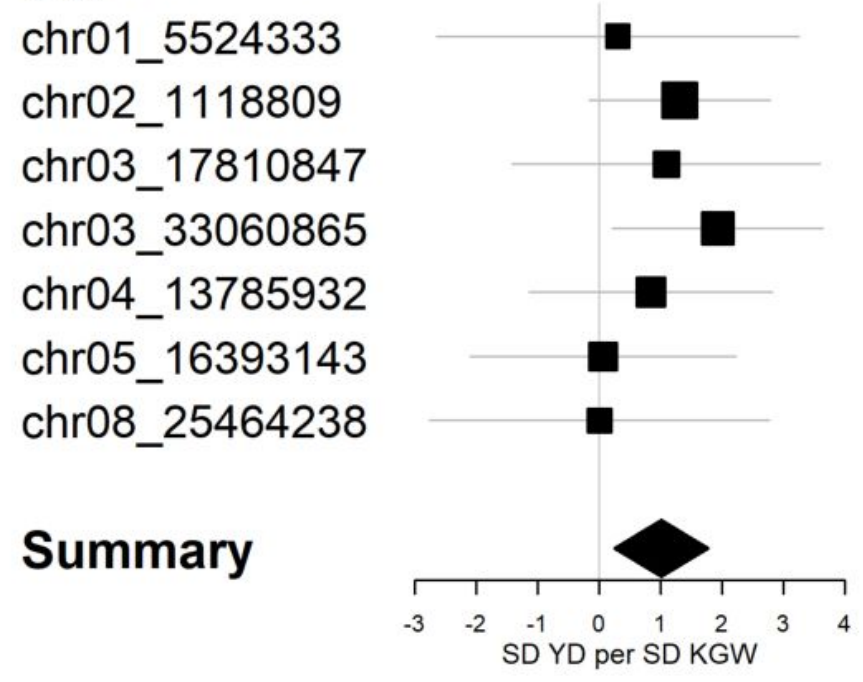

SNP

chr01_3547491

chr02_334316

chr02_7792121

chr07_23215227

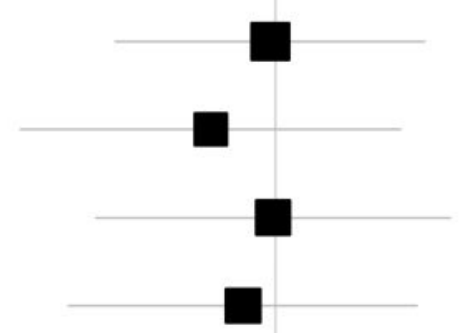

Summary

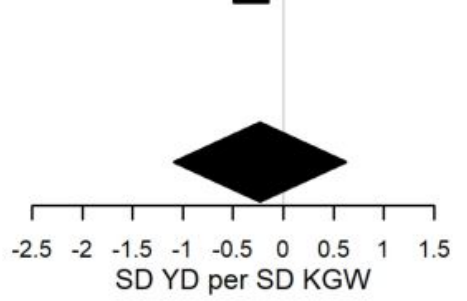

\section{Figure 3}

Genetic effect estimates of KGW and YD. Estimates are derived from IVW method of MR analyses. a Positive effect estimates between KGW and YD. b Negative effect estimates between KGW and YD. 
a

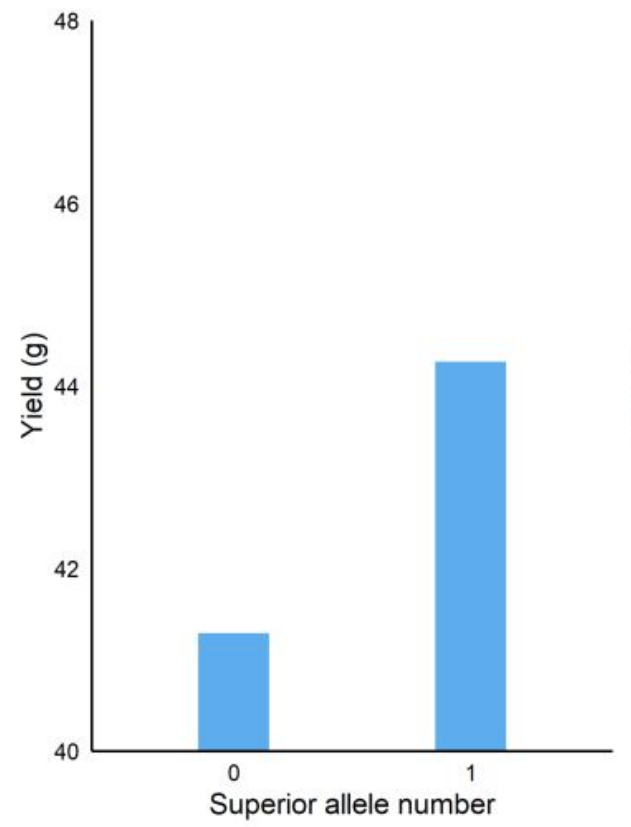

b

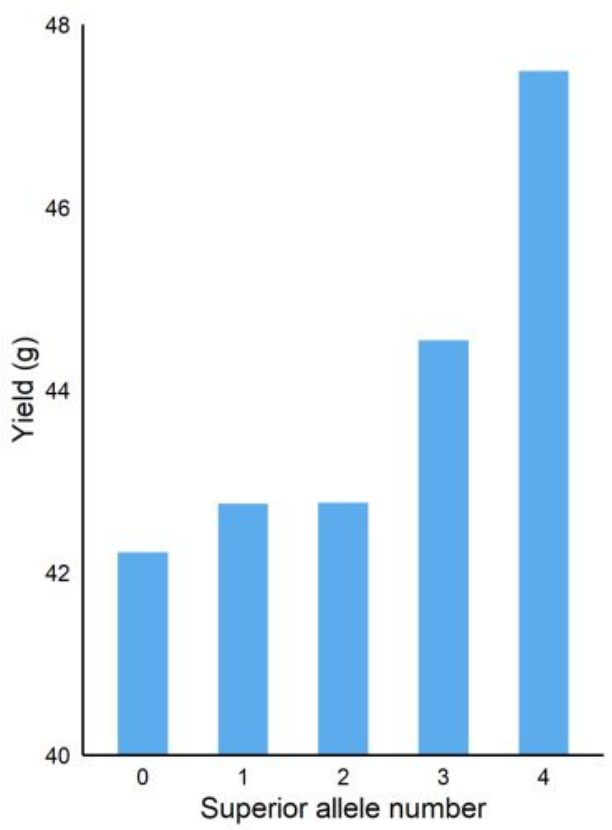

$\mathrm{c}$

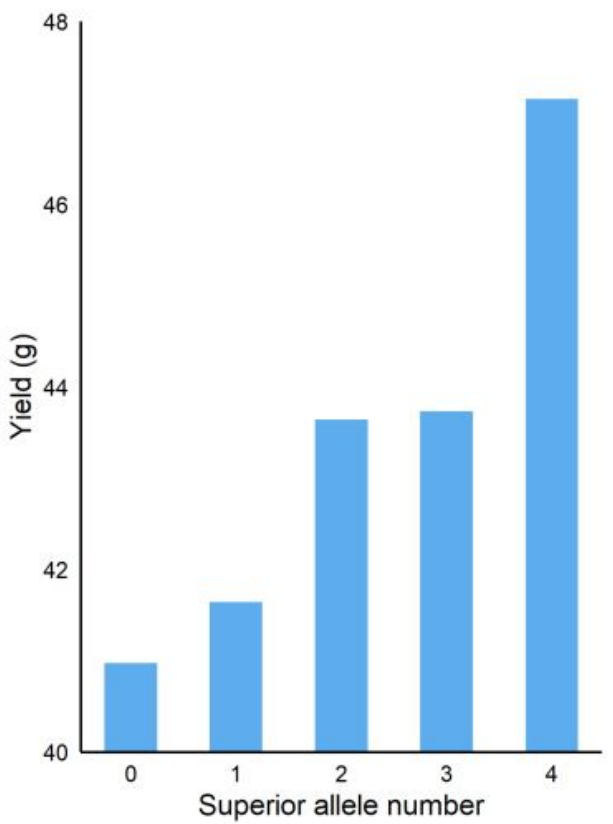

\section{Figure 4}

The average yield performance of F1 lines with different superior allele number of significant loci. a Direct loci, b Indirect loci, c Direct plus indirect loci

\section{Supplementary Files}

This is a list of supplementary files associated with this preprint. Click to download.

- Additionalfile2.xIsx

- Additionalfile1.pdf 